\title{
PERFIL FAMILIAR Y DE CONSUMO DE DROGAS EN MUJERES INTERNAS EN CENTROS DE RECUPERACIÓN EN EL NOROESTE DE MÉXICO
}

FAMILY AND DRUG USE PROFILE OF WOMEN ADMITTED IN RECOVERY CENTERS IN NORTHWEST MEXICO

Elba Abril-Valdez

Centro de Investigación en Alimentación y Desarrollo, A.C. abril@ciad.mx

Rosario Román Pérez Centro de Investigación en Alimentación y Desarrollo, A.C.

María José Cubillas Rodríguez Centro de Investigación en Alimentación y Desarrollo, A.C.

Sandra Elvia Domínguez Ibañez Centro de Investigación en Alimentación y Desarrollo, A.C.

Cómo citar este texto:

Abril-Valdez, E., Román Pérez, R., Cubillas Rodríguez, M.J. y Domínguez Ibañez, S.E. (2022). Perfil familiar y de consumo de drogas en mujeres internas en Centros de recuperación en el Noroeste de México. Health and Addictions / Salud y Drogas, 22(1), 26-39. doi: 10.21134/haaj.v22i1.600 
Abril-Valdez, E., Román Pérez, R., Cubillas Rodríguez, M.J. y Domínguez lbañez, S.E.

\section{Resumen}

Las adicciones son un problema social y de salud pública cuyas últimas tendencias en Latinoamérica evidencian un incremento en las mujeres. El objetivo de este estudio fue comparar las variables familiares y el consumo de sustancias nocivas, de mujeres internas en centros especializados del noroeste de México. El estudio fue descriptivo, con una muestra por conveniencia de 112 mujeres de 8 centros de recuperación. Se aplicó un cuestionario sobre historia de adicción y factores sociofamiliares. Los resultados no muestran que las variables familiares difieran significativamente entre los grupos formados de acuerdo con la edad de inicio del consumo. Sin embargo, quienes iniciaron antes de los 18 años de edad, en su mayoría provenían de hogares monoparentales, con padre/madre de menor escolaridad y consumo en familiares cercanos. Se concluye que por la heterogeneidad de los antecedentes, no fue posible obtener diferencias estadísticamente significativas entre el perfil familiar y las edades de inicio de la adicción. No obstante, tal diversidad permite entrever la necesidad de considerar los contextos al diseñar estrategias de prevención y atención.

\section{Abstract}

Addictions are a social and public health problem whose latest trends in Latin America show an increase in women. The objective of this study was to analyze the relationship between family variables and substance use internal women in specialized centers in northwestern Mexico. The study was descriptive, with a convenience sample of 112 women from 8 recovery centers. A questionnaire on addiction history and sociofamily factors was applied. The results did not show that family variables differed significantly between the groups formed according to the age of onset of consumption. However, those who started before 18 years of age were mostly from single-parent households, with parents with less schooling and consumption in close relatives. It is concluded that due to the heterogeneity of the background, it was not possible to obtain statistically significant differences between the family profile and the age of onset of addiction. Nevertheless, such diversity suggests the need to consider the contexts when designing prevention and care strategies.

\section{Palabras clave}

Adicciones, centros de recuperación, familia, mujeres.

\section{Keywords}

Addictions, family, recovery centers, women. 


\section{INTRODUCCIÓN}

El tema de las adicciones en las últimas décadas, al haberse hecho visible y cuantificable los estragos para la salud, se ha convertido en un problema de salud pública. Diversos estudios han documentado el impacto que tiene esta problemática en el desarrollo social, y en general, los efectos negativos que tiene y desencadena, el padecer algún tipo de adicción para las mujeres y los hombres (Pérez \& Correa, 2011; Velasco, Hernández, Herrero, Hernández, 2013; ENCODAT, 2017; Serrano, 2019).

Los efectos para la salud son múltiples. Las personas con adicción a algún tipo de sustancia están expuestas a un riesgo mayor de sobredosis mortal y se ven afectadas de manera desproporcionada por enfermedades/infecciones de transmisión sanguínea como el VIH y la hepatitis C; además del deterioro físico y mental (UNODC, 2019). Lo anterior conlleva a un incremento en el número de muertes y años de vida sana perdidos atribuidos al consumo de drogas.

Datos del Informe Mundial sobre Drogas (UNODC, 2019) señalan que, en 2017, 5.5\% de la población mundial (271 millones de personas) de 15 a 64 años consumieron una sustancia ilícita por lo menos una vez en el último año. Se estima que el número de personas que consumen drogas en la actualidad ha aumentado en un $30 \%$ con respecto a 2009, año en el que 210 millones de personas habían consumido drogas en el año anterior.

La Encuesta Nacional de Adicciones (ENCODAT, 2017) México presenta, en la población de 12 a 65 años, una tendencia creciente y sostenida en el consumo de drogas. Para el estado de Sonora la edad de inicio de consumo de drogas es de 17.8 años con diferencias por sexo de 17.7 para el caso de los hombres y 18.2 para el de las mujeres, similar a lo que se marca a nivel nacional. También refiere que de las personas con posible dependencia a drogas solo $20.3 \%$ ha recibido tratamiento. Ello significa que la mayoría, una vez que inicia, es altamente probable se mantenga en tales condiciones, situación preocupante, principalmente en las mujeres donde solo se atiende con tratamiento $12.8 \%$, porcentaje que en los hombres es de $22 \%$. En la población joven de 12 a 17 años de edad, los porcentajes son menores y las diferencias por sexo mínimas (3.4\% en hombres y $2.3 \%$ en mujeres de consumo en el último año). Si bien el consumo registrado desde hace varios años es menor en las mujeres, no deja de ser de interés para quienes generan políticas públicas, el hecho de que en las instancias de atención de esta problemática, se reporten incrementos en la solicitud de servicios de mujeres consumidoras de drogas legales e ilegales ¿Qué es lo que está pasando con las mujeres en el estado de Sonora, México para que aumenten su consumo de sustancias adictivas legales o ilegales?

Las adolescentes están consumiéndolas por primera vez alrededor de los 13 años de edad y según la ENCODAT (2017), pertenecen a familias donde no se establecen de límites claros, se carece de una autoridad democrática, hay maltrato, violencia física y emocional, sin que esto represente un prejuicio hacia las dinámicas familiares. La literatura señala que es en la familia donde se crean las bases para el desarrollo de hábitos de vida, formas de expresar afectos, relacionarse con los demás, de resolver conflictos y de desarrollar conductas de autocuidado. Así, este vínculo familiar puede tener un efecto positivo o negativo en la incidencia de conductas de riesgo tales como consumo de drogas, autoagresión, violencia, depresión entre otras (Valenzuela, Ibarra, Zubarew, et al, 2013; Perasso, Carraro \& Ozturk, 2020). Para Pérez \& Sierra (2007) y Pérez \& Correa (2011), la adicción en mujeres adultas es una manera de evadir problemas o acontecimientos estresantes provocados por situaciones familiares tales como separación de hijos, ruptura o conflictos de pareja, entre otros. Señalan que estos conflictos relacionados con su papel de madre, esposa, pareja y ama de casa y los sentimientos de frustración a estos roles, están relacionados con desarrollar de algún tipo de adicción.

to que sí-También se ha documentado es que el género es un determinante de la salud y como tal constituye un elemento clave para comprender los distintos significados que el uso y abuso de drogas tienen para las mujeres al igual que las creencias asociadas. Asimismo, permite conocer y analizar las motivaciones e influencias que las impulsan a iniciarse en el consumo y a desarrollar tales comportamientos. Los modelos sociales de masculinidad y feminidad que se concretan en roles y normas de género, fijan la aceptabilidad o el rechazo de ciertas conductas para los hombres y las mujeres. Estas normas de género ejercen una gran influencia en las relaciones que los hombres y las mujeres mantienen con las drogas. Mientras entre los hombres el consumos de drogas legales pueden ser hasta cierto punto socialmente aceptados, entre las mujeres es un reto a los valores dominantes. Las mujeres suelen ser 
Abril-Valdez, E., Román Pérez, R., Cubillas Rodríguez, M.J. y Domínguez Ibañez, S.E.

estigmatizadas y reciben menor grado de apoyo familiar o social (Sánchez, 2009; Medina Mora et al., 2012).

Frecuentemente, los estereotipos vinculados a los roles femeninos tradicionales, disparan el uso de ciertas drogas, como es el caso de los psicofármacos, sustancias que se consideran compatibles con las necesidades y problemáticas femeninas; tales como la depresión, la ansiedad y ciertos trastornos psiquiátricos (Romo \& $\forall$ Gil, 2006). Esta dimensión individual ayuda a entender por qué las motivaciones de las mujeres para consumir drogas se vinculan en mayor medida como una respuesta a estados de ánimo negativos, mientras que entre los hombres prevalecen las motivaciones relacionadas con la interacción social.

A pesar de que las estadísticas expresan un consumo menor en las mujeres a diferencia de los varones, éstas son más susceptibles de desarrollar una adicción y más vulnerables a sus efectos y tienen mayor riesgo de sufrir violencia física y sexual. Las mujeres con problemas de abuso o dependencia a las drogas se enfrentan a estigmas culturales y múltiples barreras que les impiden acudir a los servicios asistenciales y tener mejores resultados en los programas de tratamiento. Actualmente, la mayoría de las estrategias para la prevención de las adicciones no consideran las diferencias de género y toman como referentes básicos las necesidades de los varones (Sánchez, 2014).

Estudios epidemiológicos realizados a nivel nacional en los Centros de Integración Juvenil (CIJ), muestran un incremento en el número de mujeres que acuden a tratamiento por problemas relacionados con el uso de sustancias, al pasar de una mujer por cada 12 hombres en 1990 a una mujer por cada cinco hombres en 2017. En este último año, más del 50\% de las mujeres acudió personalmente a solicitar tratamiento a la edad promedio de 22.6 años, la mayoría eran solteras, con estudios de secundaria y se dedicaban principalmente a estudiar o labores del hogar (Benítez, 2018). De ahí la pertinencia de estudiar la relación de las variables familiares con consumo temprano de sustancias nocivas, objetivo del presente trabajo cuya metodología se presenta a continuación.

\section{METODOLOGÍA}

El estudio fue descriptivo de corte trasversal. Se eligió trabajar con la población femenina atendida en los Centros de Rehabilitación de Adicciones, por considerar que se trata de mujeres que de alguna manera han reconocido su adicción por las sustancias, ya sea por sí mismas o impulsadas por familiares o autoridades que las condicionan para así permitirles interactuar con sus hijos o hijas. Si bien tal situación puede dar lugar a una perspectiva distinta a la de quienes consumen fuera de éstos, su internamiento facilitó establecer el contacto y previa autorización de ellas, entrevistarlas durante el proceso de rehabilitación una vez que han sido desintoxicadas.

Los Centros fueron elegidos por tener el reconocimiento del Consejo Estatal contra las Adicciones del Estado de Sonora (CECA) y cuyos datos nos fueron proporcionados por la Dirección de Salud Mental de la Secretaría de Salud, además de ser los que atienden a población femenina. Se incluyeron Centros de tres localidades: Hermosillo (8), Nogales (1) y Ciudad Obregón (1), ciudades que se encuentran entre los diez principales asentamientos urbanos del estado y geográficamente distribuidos en las zonas norte, centro y sur.

\section{Participantes}

La integración de la muestra se hizo por conveniencia a partir de la disponibilidad (Padua, 1996). Se trabajó con 112 mujeres, que fue el total que se encontraba en condición de internamiento en los diferentes centros de rehabilitación en adicciones, el día de las visitas agendadas a los centros y con disponibilidad para responder el instrumento diseñado para este estudio. Como criterio de exclusión, se consideró a aquellas mujeres de ingreso reciente ya que deben pasar por una fase de desintoxicación física y psicológica de sustancias que altera su estabilidad física y emocional.

\section{Instrumento:}


Se aplicó un cuestionario adaptado para el estudio que incluyó variables atributivas, tales como el sexo, escolaridad, tipo de familia, entre otras. Asimismo, se obtuvieron indicadores sobre patrones de consumo y algunos indicadores de la dinámica familiar. Para cumplir con los criterios de precisión y generalización de los resultados, el instrumento se conformó a partir de cuestionarios ya validados en población similar al grupo objetivo para asegurar la confiabilidad y validez de los datos.

El cuestionario se conformó por 12 apartados, pero para los fines este artículo, solo se retomaron cuatro relacionados con el contexto familiar, los cuales se describen a continuación:

Para caracterizar a las participantes se pidió información sobre: edad, escolaridad, estado civil, ocupación y número de hijos. En antecedentes familiares se incluyó presencia o ausencia de padre/madre, escolaridad de él/ella, dinámica conyugal para la solución de conflictos y nivel económico familiar considerando la suficiencia o insuficiencia de ingresos para cubrir gastos básicos tales como si les alcanzaba para comprar comida todos los días, pagar deudas, comprar medicinas, entre otros gastos.

Para consumo de sustancias se utilizó la herramienta ASSIST, por sus siglas en inglés, (Alcohol, smoking and substances involvement screening test) validado al español por la Organización Mundial de la Salud (WHO, 2002 ). Se trata de un cuestionario breve para identificar los niveles de uso perjudicial de sustancias que contiene ítems sobre el consumo reciente y a lo largo de la vida de 10 sustancias (tabaco, alcohol, cannabis, cocaína, anfetaminas u otros estimulantes, ansiolíticos, alucinógenos, inhalantes y otras drogas). Asimismo, contiene preguntas sobre edad de inicio de consumo, razones para el uso de drogas, percepción de la disponibilidad de las sustancias en distintos contextos, consumo en el espacio familiar y social, en familia y pareja, influencia de amigos /o pareja en el consumo, además de exposición a la prevención y tratamiento por consumo, entre otras.

\section{Procedimiento}

Durante los meses de septiembre y octubre de 2018 se visitó cada lugar y se explicó al encargado el objetivo de estudio para obtener autorización y poder aplicar el cuestionario. Se eligieron horarios en los que hubiera la menor interferencia con las actividades programadas en cada Centro. Las mujeres eran reunidas en un espacio proporcionado por el/la encargado/a, generalmente alguna aula que utilizaban para realizar sus actividades.

Antes de iniciar, se explicaba a las mujeres el objetivo del estudio y la importancia de su participación. Se-solicitaba la firma del formato de consentimiento informado, incluido en la primera página del cuestionario, en el cual se enunciaba de manera explícita el carácter de confidencialidad y anonimato de la información proporcionada, tanto para la participante como para la institución. Igualmente se asentaba su aceptación de participación voluntaria en el estudio. Posteriormente se proporcionaban las instrucciones precisas sobre la respuesta que se esperaba de la persona participante, a fin de posibilitar que la información fuera lo más veraz y apegada a la realidad. El cuestionario fue auto aplicado, sin embargo, en algunas ocasiones fue necesario hacerlo de manera personal dada la dificultad de comprensión de lectura de algunas participantes. La duración aproximada para responderlo osciló entre 30-40 minutos.

\section{Análisis de datos}

La información fue procesada en una base de datos y analizada con el software estadístico IBM-SPSS versión 18. Se utilizó análisis descriptivo para generar los perfiles demográficos, familiar, escolar, laboral de las participantes lo cual nos permitió caracterizar las condiciones de vida de este grupo de población.

Para la comparación de las variables familiares y la historia de consumo de drogas, la muestra se dividió en tres grupos de acuerdo con la edad de las participantes. El de adolescentes: de 14 a 19 años (n=17); de jóvenes: de 20 a 29 años $(n=54)$ y de adultas: de 30 años y más $(n=33)$. Los resultados se presentan iniciando con el perfil sociodemográficos de las participantes, seguidamente la historia del consumo de sustancias por rango de edad, y posterior- 
mente se exponen las diferencias en variables familiares asociadas al consumo temprano. Para consumo temprano se forman dos grupos las que iniciaron antes de los 18 años y las que lo hicieron después de esa edad. Dado que la información en la mayor parte de las variables era de tipo categórica, se realizó la prueba no paramétrica \2 para la comparación de proporciones entre los grupos, mismos que se describen a continuación

\section{RESULTADOS}

El promedio de edad de las participantes fue de 27.4 años con un rango de 14 a 50 años de edad. El 15.3\% tenían entre 14 y 19 años, $48.6 \%$ entre 20 y 29 años y 29.7 entre 30 y más de edad. Más de la mitad (60.2\%), reportó estar soltera y et $32.4 \%$ dijo tener pareja (casada/unión libre). La media de escolaridad para este grupo fue de 9.33 años de escolaridad equivalente al nivel de secundaria, promedio alcanzado en el estado de Sonora. Cabe señalar que 94.4\% no estaba estudiando al momento de ingresar al centro, en el rango de 14 a 19 años ninguna dijo haber dejado haber dejado la escuela para ingresar al centro, lo que nos indica abandono escolar temprano. La mayoría de las mujeres (74.5\%) reportó tener hijos(as), en un rango que va de 1 a 11 y de estas sólo 46\% vivía con ellos antes de su internamiento.

En relación a la ocupación laboral, 22.7\% dijo tener algún tipo de ingreso por concepto de su trabajo, antes de ingresar al centro. La mayoría (54.9\%) se desempeñaba como empleada del sector comercio, 15.2\% trabaja en la limpieza de casas, el resto laboraba en actividades tales como: ventas por teléfono o de artesanías, entre otras. La situación económica de las participantes, se evaluó a través de una serie de preguntas que determinan la capacidad de gasto en las familias, en función de si tienen dinero para cubrir sus necesidades básicas. Ante los indicadores presentados, respondieron con las opciones algunas veces o nunca: se tenía dinero en casa para la compra de comida (86\%), ropa (62\%), pagar las cuentas/deudas (70\%), útiles escolares (75\%), y atender problemas de salud (78\%). Lo anterior evidencia que, en estas familias, los ingresos no les alcanzan para pagar los gastos mínimos de la sobrevivencia

En cuanto a situación familiar et $53 \%$ de las participantes dijo contar con la presencia de ambos padres y et $47 \%$ con uno de ellos o ninguno. Se les preguntó sobre la relación de los padres, et $80 \%$ señaló que discutían cuando estaban juntos y que no sabían resolver sus problemas. Igualmente señalaron la presencia de golpes (42\%) e insultos (67\%). En muestras de afecto, $70 \%$ señaló observar muestras de afecto entre ellos y $75 \%$ buscar tiempo para pasarla juntos.

Historia de consumo de sustancias y rango edad

Considerando la heterogeneidad de las participantes el análisis se realizó a partir de tres grupos de edad: 14 a 19 años (adolescentes), de 20 a 29 años (jóvenes) y de 30 y más (adultas). Esto con el fin de poder realizar un análisis más detallado dado que las condiciones de consumo según la edad pueden ser muy diferentes entre grupos.

La media de edad de inicio de consumo para el grupo de adolescentes fue de 14 años con un rango de edad de 13 a 16 años. En el grupo de jóvenes la media fue de 18.72 años con un rango de 12 a 28 años; para las adultas la media de edad fue 23.92 años con un rango de 13 a 38 años. En los tres grupos se encontró contacto temprano con las sustancias nocivas legales e ilegales.

En relación al consumo de sustancias, las principales drogas de consumo diario mencionadas por los grupos fueron el tabaco, las anfetaminas como el cristal y otros derivados, la marihuana y los sedantes (Gráfica 1). Por otro lado, no son usuarias de una solo sustancia, sino poliusuarias, en todos casos se reportó usar más de una droga. Cabe destacar que la heroína y el M30 (Fentanilo, narcótico usado como analgésico y anestésico, opiáceo más potente disponible para uso médico), aunque con menor porcentaje, también aparecen como sustancias de consumo diario en el caso de las jóvenes. Por otro lado, el alcohol fue mencionado en mayor porcentaje su consumo entre las jóvenes y las adultas. La marihuana y los inhalantes se reportan con mayor frecuencia entre las adolescentes. 
Gráfica 1. Porcentaje de consumo diario de sustancias

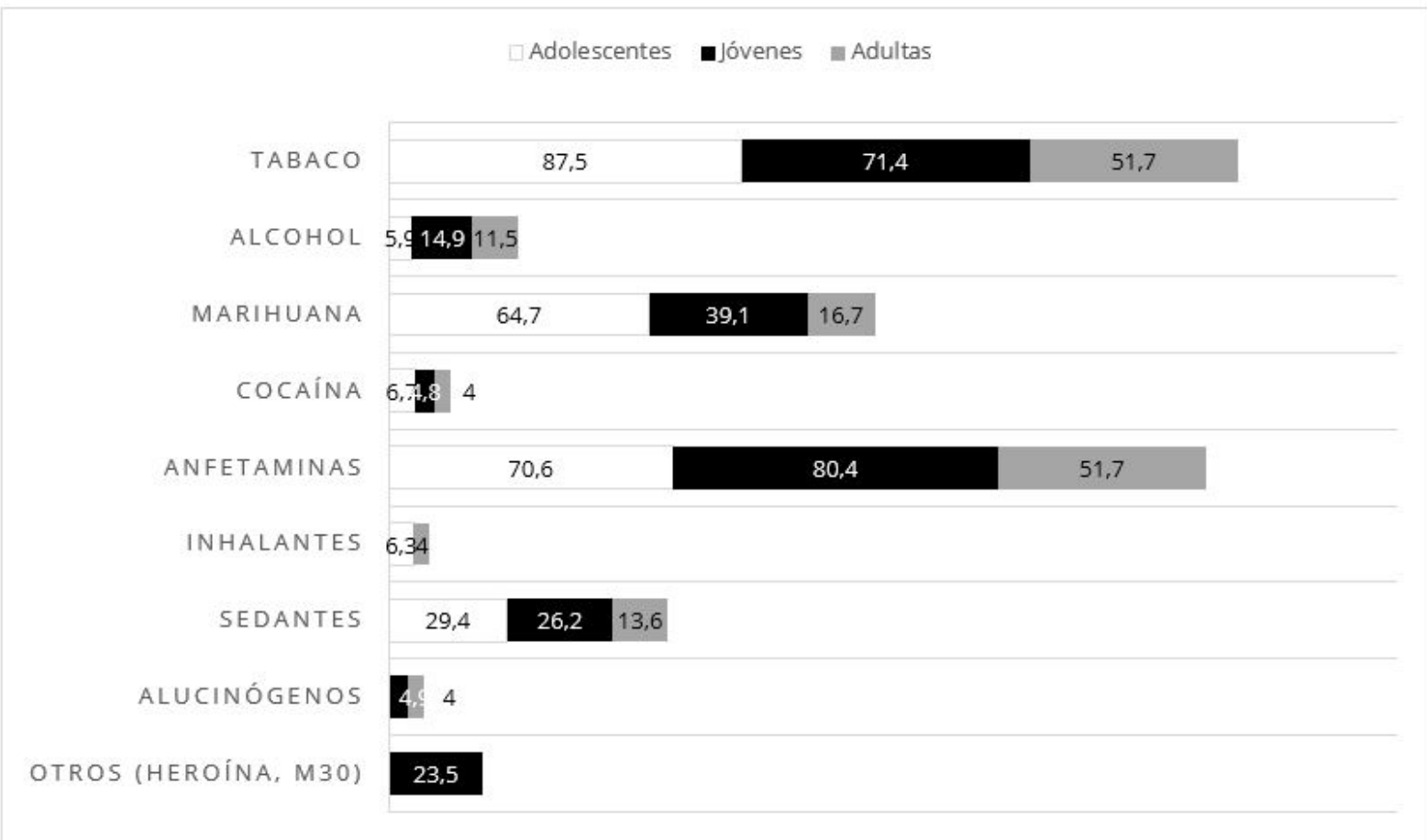

Fuente: elaboración propia

El $58 \%$ de las adolescentes y $66 \%$ de jóvenes y adultas reportaron el uso de algunas sustancias como apoyo para bajar o subir de peso, igualmente son usadas para tratar de tener más energía. Para explorar este aspecto se les preguntó si las habían utilizado sin que un médico se las recetara. En los tres grupos se mencionó haber usado/ probado algún tipo de anfetamina o estimulante como apoyo para controlar el peso o tener más energía sin supervisión médica ( $59 \%$ de las adolescentes, $63 \%$ de las jóvenes y $60 \%$ de las adultas). En cuanto al uso de tranquilizantes para calmar los nervios o dormir, tales como el Librium, Valium, Diazepam entre otros, $58.8 \%$ de las adolescentes, $63 \%$ de las jóvenes y $30 \%$ de las adultas dijo haberlos tomado sin que un médico se los haya prescrito. Esto datos evidencian el uso a temprana edad de sustancias ilegales, lo que aumenta la probabilidad de desarrollar algún tipo de adicción.

El acceso fácil a las sustancias legales e ilegales es una situación que debe ser analizada con mayor profundidad ya que muestra la disponibilidad que cualquier persona puede tener para consumir ese tipo de medicamentos sin una receta. El cuestionario incluyó una pregunta sobre dónde conseguían las drogas que consumían y las respuestas incluyeron la escuela, el trabajo o la colonia. Destaca que, en cualquier escenario, en mayor o menor proporción, hay disponibilidad de drogas. En los tres grupos consideran que en la colonia es más fácil conseguir droga (93.4\% de las adolescentes, $79.8 \%$ de las jóvenes y $73.8 \%$ de las adultas) que en el trabajo (50\% de las adolescentes, 22.2 de las jóvenes y $18.2 \%$ de las adultas) o escuela (31\% de las adolescentes, 22.2 de las jóvenes y $6 \%$ de las adultas).

Al explorar las razones de inicio en el consumo la diversidad de respuestas muestra que; en las adolescentes, principalmente son desde la curiosidad, evadir problemas en casa, imitar a los amigos hasta aquellas que tienen que ver con la búsqueda de la tranquilidad y el sentirse bien. En el grupo de jóvenes y adultas destacan la curiosidad y escapar de problemas de casa, así como complacer a la pareja. Cabe destacar que en el grupo de adolescentes y adultas la depresión aparece con mayor porcentaje que en las jóvenes como razón para el consumo (Gráfica 2).

Gráfica 2. Razones para consumo de sustancias por primera vez 
Abril-Valdez, E., Román Pérez, R., Cubillas Rodríguez, M.J. y Domínguez Ibañez, S.E.

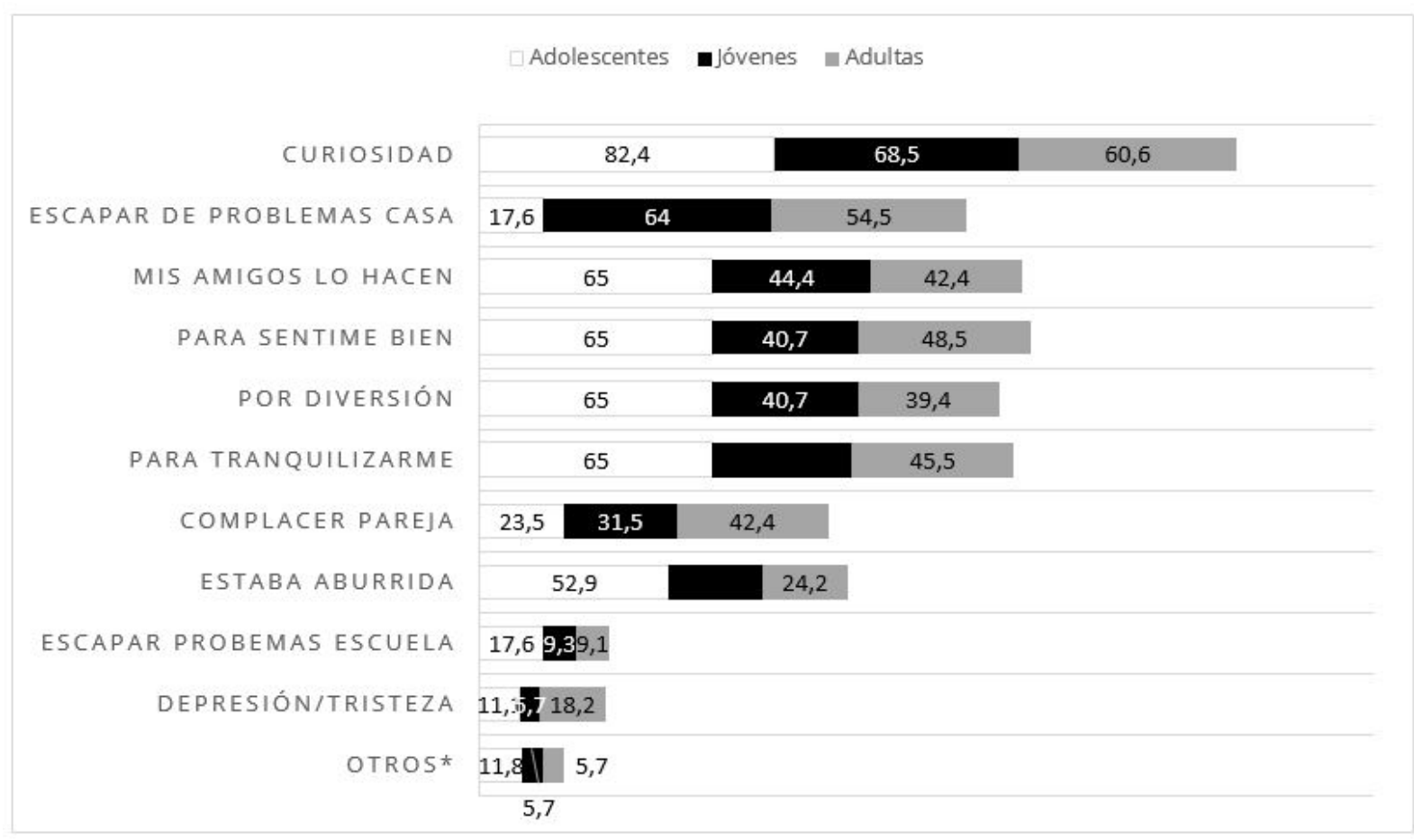

Fuente. Elaboración propia

* Otros (Llamar atención de padres, bajar de peso, para tener sexo diferente, por el trabajo, la droga ya no me hacía, yo vendía)

**La suma de porcentajes excede el $100 \%$ ya que posible elegir más de una opción

En relación con quien les proporcionó la droga la primera vez, en los tres grupos la figura de los/las amigos/as aparece como uno de los principales proveedores o facilitadores. En el caso de las adolescentes $82.4 \%, 64.8 \%$ de las jóvenes y en las adultas $60.6 \%$ dijo que un amigo/conocido se las facilitó por primera vez. La pareja también fue mencionada en mayor proporción entre las adultas (45.5\%) que en los demás grupos (23.5\% de las jóvenes y 27.8 de las jóvenes). Cabe destacar cómo el hogar también es un facilitador de las sustancias, $23.5 \%$ de las adolescentes, $13 \%$ de las jóvenes y $9.1 \%$ de las adultas dijeron que un familiar había sido quien les dio acceso.

En el consumo subsecuente los amigos o conocidos ( $58.8 \%$ adolecentes, $46.3 \%$ jóvenes y $45.5 \%$ adultas) y vendedor de droga (52.9\% adolescentes, $44.4 \%$ jóvenes y $45.5 \%$ adultas) fueron mencionadas como la principal fuente para proveer la sustancia en ambos grupos antes de ingresar al centro. Cabe mencionar que entre las jóvenes (31.5\%) y adultas (39.4\%) aparece también, en mayor proporción la pareja como principal proveedor subsecuente, menos que en las adolescentes (17.6\%). La familia como facilitadora subsecuente aparece en mayor proporción entre las adolescentes (23.5\%) más que entre las jóvenes (11.1\%) y adultas (6.1\%). Cabe señalar que no es solo un proveedor al que acuden, sino que puede ser una combinación de varios, lo que ratifica la facilidad de acceso a las sustancias ilegales en los contextos de las participantes.

A fin de documentar esa posibilidad, se preguntó quién de las personas que conocían acostumbraban consumir sustancias. Los datos muestran que, en los tres grupos, era común que las participantes mantuvieran relación con más de una persona consumidora de drogas. Dentro de su círculo familiar mencionaron a la madre y el padre, así como a los hermanos con algún tipo de adicción. Por otro lado, también la pareja aparece como consumidor dentro de su grupo cercano (Gráfica 3). Incluso en el grupo de adultas se reporta tener hijos que consumen algún tipo de droga. Los datos podrían indicar que las participantes interactúan en un ambiente familiar en donde el consumo de drogas está quizá normalizado lo cual facilita las recaídas y dificulta su rehabilitación. El consumo por parte de los padres y otras personas cercanas, no sólo afecta el empleo de las mismas sustancias en jóvenes, sino que también puede incrementar la probabilidad de utilizar otras sustancias e incluso de llegar al policonsumo. 
Gráfica 3. Personas cercanas que usan algún tipo de droga

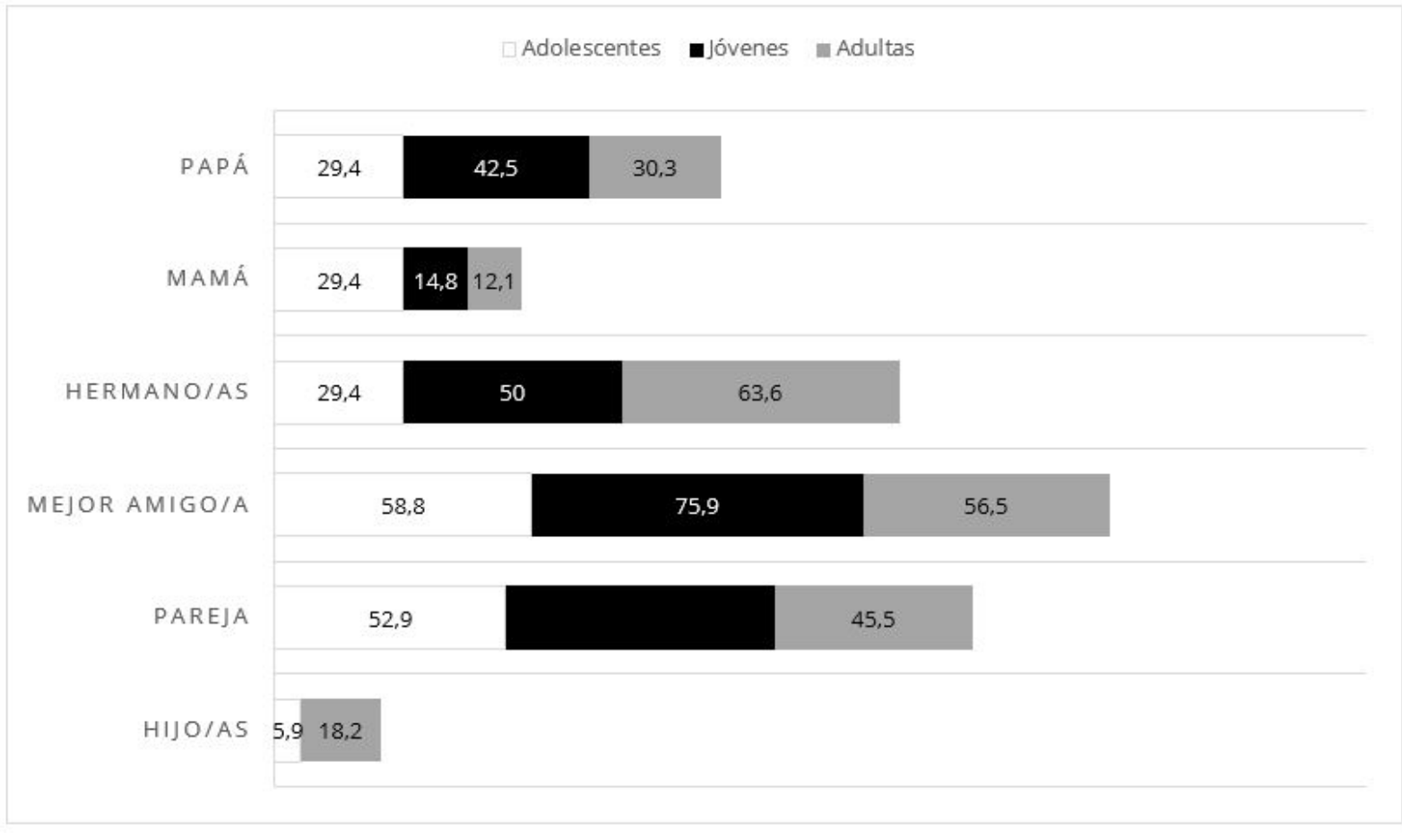

* La suma de porcentajes excede el $100 \%$ ya que posible elegir más de una opción

Los y las amigas, así como la pareja pueden constituirse en una influencia importante en el inicio y progreso del uso de drogas, además de ser uno de los principales proveedores de sustancias. La presión ejercida por el grupo de pares para incitar el consumo puede ser uno de los principales factores de riego que aumenta la probabilidad de que se presente la conducta. Entre las participantes aun cuando más de la mitad en los tres grupos (47\% adolescentes, $51 \%$ jóvenes y $60 \%$ de las adultas) reportó no haber sentido presión por parte de sus amigos para iniciarse en el consumo, es importante señalar el porcentaje que en mayor o menor proporción (poco más del $40 \%$ en los tres grupos) sí sintió esa presión por parte de los amigos cercanos, principalmente en el grupo de adolescentes (53\%). Así la necesidad de ser aceptado por el grupo puede llevar a ceder a la presión quedando en una situación de vulnerabilidad, lo que ya ha sido documentado en otros estudios (Calvete \& Estevéz, 2009; Hernández, Font \& Gras, 2015).

En cuanto a la influencia de la pareja, $30 \%$ de las adolescentes, $38 \%$ de las jóvenes y $32 \%$ de las adultas participantes dijo haber sentido entre poca a mucha presión de su novio/pareja para que consumiera algún tipo de droga. Esta situación las coloca en riesgo, pues el temor a ser abandonas o rechazadas por su pareja las lleva a aceptar condiciones con las que no están de acuerdo como puede ser el uso y abuso de sustancias

Por lo que se refiere a procesos de recuperación, las participantes son mujeres que han sido tratadas más de una vez por su adicción y han sido protagonistas de más de una recaída, incluso algunas más de 6 veces. Otras han asistido a Centros de consulta externa y grupos de apoyo (Tabla 1).

Tabla 1. Proceso de recuperación

\begin{tabular}{|c|c|c|c|c|c|}
\hline \multirow[t]{3}{*}{ Situación } & & & Jóvenes & \multicolumn{2}{|c|}{ Adultas } \\
\hline & \multicolumn{2}{|c|}{ Adolescentes } & Fr. $\%$ & Fr. & $\%$ \\
\hline & Fr. & $\%$ & & & \\
\hline Ha tenido una sobredosis de droga más de una vez & 10 & 62.5 & $18 \quad 33.4$ & 8 & 23.2 \\
\hline Ha sido tratada por abusar de las drogas más de una vez & 10 & 62.5 & 3564.9 & 15 & 45.4 \\
\hline
\end{tabular}


Abril-Valdez, E., Román Pérez, R., Cubillas Rodríguez, M.J. y Domínguez Ibañez, S.E.

Ha asistido a lugares de tratamiento de consulta externa por tu consumo de drogas más de una vez

Has asistido a grupos de auto ayuda como AA o drogadictos

6

37.5

$6 \quad 37.6$
3055.5

3055.5
$13 \quad 39.4$

$10 \quad 30.3$

anónimos más de una vez

Fuente: Elaboración propia

En este punto es importante señalar las menores de 19 años que señalaron haber tenido una sobredosis de la sustancia consumida (62.5\%), al igual que 62.5\% tiene antecedentes de tratamientos anteriores por abusar o depender alguna.

Factores familiares asociadas al consumo temprano de sustancias

Con relación a factores familiares que pudieran influir en el inicio temprano de consumo de drogas, se analizó la relación entre la presencia de ambos padres, la escolaridad de los padres y el consumo de sustancias por padres u otro miembro de la familia o la pareja en el caso de tenerla (Tabla 2). Considerando que la literatura en México señala que en las mujeres la edad de inicio en el consumo de drogas es a los 18 años, se tomó esa edad como punto de corte para el análisis de consumo temprano (ENCODAT, 2017; UNODC, 2019). Los resultados muestran que no existe una asociación estadísticamente significativa entre la presencia de ambos padres o uno de ellos y la edad de inicio para el consumo de drogas $\left(X^{2}(1)=1.333, p=.184\right)$. Sin embargo, cabe destacar que fue mayor la proporción de participantes que iniciaron en el consumo antes de los 18 que señalaron la presencia de solo uno de los padres.

Tabla 2. Distribución porcentual de variables familiares asociadas al inicio temprano de consumo de drogas

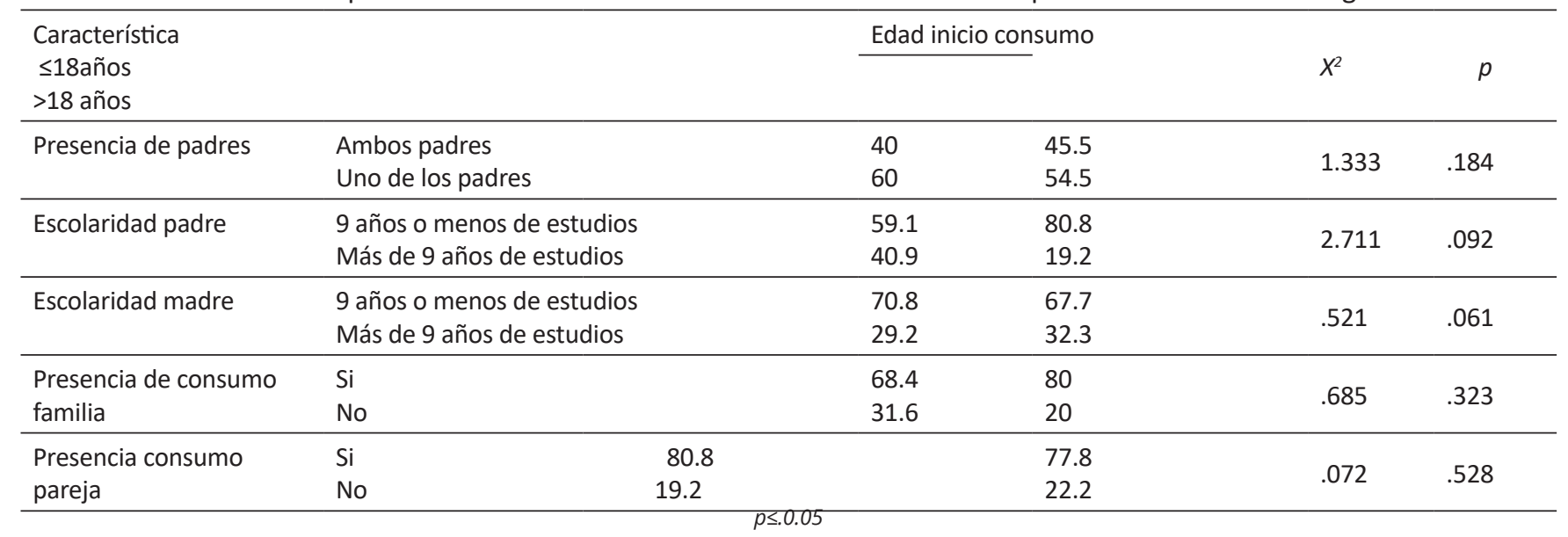

Fuente: Elaboración propia con datos de encuesta

En cuanto a los años de escolaridad de los progenitores, los datos muestran que a más años de escolaridad del padre y de la madre, la edad de inicio de consumo de drogas se retrasa un poco. Si bien esta asociación no resultó estadísticamente significativa, se observó que las mujeres con inicios de consumo en mayor edad, sus padres y madres tenían más de nueve años de estudios $\left(X^{2}(1)=2.711, p=.0 .92\right.$ vs $\left(X^{2}(1)=0.521, p=.061\right)$. Esta misma tendencia aparece en relación a la adicción o consumo de padres, hermanos o pareja. Por el contrario, el consumo temprano es reportado por las mujeres cuando hay adicciones en el círculo familiar cercano (Tabla 2).

\section{Discusión}

Los datos de consumo reportados por las participantes concuerdan con los del Informe Mundial sobre las Drogas 
(UNODC, 2019) al encontrar que la mariguana junto con las anfetaminas y estimulantes son las sustancias más frecuentes consumidas en el mundo. Además, en ese mismo informe, se señala que los/las usuarios(as) de sustancias, sea de manera ocasional o habitual, tienden a utilizar más de una sustancia ya sea de manera simultánea o sucesivamente, dato que también aparece en este estudio independientemente del rango de edad y del perfil familiar.-

Las edades de inicio en el uso/consumo de sustancias nocivas, identificadas en los tres rangos de edad, son alrededor de los 12 años, etapas tempranas de la vida en las que generalmente las mujeres están en el último grado de la educación primaria. Coincide también con la pubertad y con ella el despertar en las mujeres de nuevas sensaciones en su cuerpo y en su vida. En esa metamorfosis femenina, los entornos familiares adquieren aun mayor relevancia de la que tradicionalmente se les ha reconocido. Como muestran los datos de este estudio, fue en el seno familiar donde muchas mujeres convivieron con modelos maternales, paternales o fraternales ligados a las adicciones. Tanto en el grupo de adolescentes como en el de jóvenes y adultas, en su entorno cercano y familiar hubo acceso a algún tipo de sustancia, así como presencia de familiares o pareja con adicción. Estos datos pudieran señalar que este tipo de comportamiento, quizá fue aprendido y normalizado en el contexto familiar, por lo que se convierte en un factor de riesgo para mujeres como las participantes, que al salir del centro de rehabilitación regresan al mismo contexto facilitador de droga, aumentando con ello su probabilidad de recaer.

Hernández, Font y Gras (2015) señalan que la observación de modelos de conducta que propician el inicio al consumo de sustancias en los y las jóvenes puede propiciar el aumento de su frecuencia. Señalan que los y las jóvenes fumadores suelen tener padre y/o madre y/o amigos también fumadores, además de que este tipo de comportamiento facilita la repetición de patrones de conducta aprendidos en la familia. Por otro lado, el inicio temprano de tabaco u otras drogas está relacionado a un consumo más prolongado y grave y por ende con más resistencia al tratamiento (Blanco, Sirvent \& Palacios, 2011)

Los y las amigos/as, así como la pareja, pueden constituirse en una influencia importante en el inicio y progreso del uso de drogas, además de ser figuras presentes, según los resultados, como uno de los principales proveedores de sustancias. La presión que se puede ejercer por el grupo de pares para incitar el consumo, puede ser uno de los principales factores de riesgo que aumenta la probabilidad de que se presente la conducta. Entre las participantes, independientemente del rango de edad, más de la mitad reportó no haber sentido presión por parte de sus amigos para iniciarse en el consumo, es importante señalar el porcentaje que en mayor o menor proporción sí sintió esa presión por parte de los amigos cercanos. Los datos permiten asumir que la influencia de pares o personas significativas requiere estudiarse más, con otro tipo de metodología. Las presiones hacia la conformidad grupal pueden actuar como elemento que sustenta formas de conducta y actitudes. Así, el compromiso con el grupo de pares supone la adopción de conductas susceptibles de satisfacer determinadas expectativas de los demás (Van Avermaet, 1992). Pérez (2011) señala que el modelado o aprendizaje social parece ser el mecanismo más importante que explica la influencia de los pares en el consumo y abuso de alcohol, tabaco y otras drogas. Así la necesidad de ser aceptado por el grupo puede llevar a ceder a la presión quedando en una situación de vulnerabilidad. En un estudio realizado con universitarios en México que concluye que a mayor presión de pares mayor son las actitudes que favorecen el consumo de drogas ilícitas. Asimismo, a mayores actitudes favorables al consumo de drogas ilícitas mayor es la probabilidad del consumo de drogas ilícitas en jóvenes universitarios (Guzmán, Vargas, Candia, Rodríguez \& López, 2018).

Los motivos señalados en los tres grupos destacan la curiosidad, la diversión, el sentirse bien hasta escapar de problemas. Los resultados muestran la variedad de factores que pueden influir en la decisión de usar drogas ilegales. Algunos autores señalan que es posible que los jóvenes decidan consumir drogas ilícitas en la búsqueda de sentirse bien, olvidar sus problemas, divertirse, satisfacer su curiosidad, sentirse adultos y demostrar su independencia. Por lo que las actitudes que los y las jóvenes construyan hacia el consumo de las sustancias es una de las variables que ha mostrado relación con el consumo de drogas ilícitas. Actitudes que generalmente se ven reforzadas por su entorno inmediato, independientemente de la edad que se tenga (Calvete \& Estevéz, 2009; Gutiérrez \& Romero, 2014; Guzmán et al , 2018).

En relación a la asociación de variables familiares y el inicio temprano de consumo de drogas, los datos señalan que en este grupo, las variables tales como la escolaridad de los padres, la presencia de ambos padres y la adicción en 
Abril-Valdez, E., Román Pérez, R., Cubillas Rodríguez, M.J. y Domínguez Ibañez, S.E.

algún familiar o pareja no aportan mayor significancia para iniciarse en el consumo de drogas antes o después de los 18 años. Sin embrago, cabe señalar que la familia juega un rol primordial para la prevención de conductas de riesgo. Así, padre y madre son fundamentales por la influencia que ejercen en el desarrollo de hábitos de vida saludables en la forma en que interactúan en su medio y solucionan sus conflictos. Dicha relación puede tener un efecto positivo o negativo en la presencia de conductas de riesgo comprobándose que a mayor apoyo y control de los padres hay menor consumo de drogas, violencia, depresión, entre otras (Cid-Monckton \& Pedrao, 2011; Valenzuela, Ibarra, Zubarew, et al, 2013).

Finalmente, cabe recordar que se trata de una muestra específica de mujeres que han sido tratadas en su adicción más de una vez, lo que puede explicar la homogeneidad de las variables familiares en las que el hogar resultó ser uno de los lugares de acceso a las sustancias. Por tanto, una vez terminado el tratamiento las recaídas son comunes al regresar a un espacio que facilita y en el peor de los casos, promueve, el consumo de sustancias ilegales. Lo anterior señala la necesidad de evaluar los programas de los Centros de Rehabilitación de mujeres y sugerir que en todos los casos, exista un seguimiento puntual de la recuperación. Galaviz (2015) señala la importancia de también analizar las características de los centros de rehabilitación y no solo el entorno de las mujeres a fin de tener un enfoque integrado de la problemática. Hace énfasis en la sensibilización ante la importancia de las diferencias de género y hacer visibles las desigualdades que definen y guían gran parte de nuestro actuar como seres sociales y que dificultan el proceso de recuperación de las mujeres.

\section{CONCLUSIONES}

El perfil de las mujeres participantes que consumen sustancias nocivas se complejizan en tanto que los rangos de edad van desde los 14 años hasta los 50, de solteras a con pareja conyugal, de sin hijos/as a con hijos/as, de sin educación formal hasta con educación universitaria, de eternas desempleadas tal como actualmente se define a las mujeres dedicadas al hogar a mujeres con empleos formales. Tampoco en las formas de consumo encontramos sistematicidad. Lo mismo algunas se apegan al esquema de tabaco y alcohol como drogas de inicio que otras empiezan con las Ilamadas "duras" como cocaína, heroína o cristal. Este menú variado de trayectorias, muestra que cualquier programa de prevención/atención no debe partir de la consideración de una población objetivo homogénea sino más bien, asumir su heterogeneidad y con ello la necesidad de más de una estrategia de prevención y atención.

La mayoría de las mujeres participantes en este estudio provenían de entornos con economías restringidas donde son las más carencias que las oportunidades, donde la facilidad para obtener sustancias nocivas habla de la falta de eficacia en los programas de las autoridades encargadas de su control. Igualmente, si se trata del sector educativo, que del de salud, desarrollo social o seguridad pública, la intervención debe ser transversal.

Los resultados del presente estudio muestran que, sin ser significativos, hay una tendencia a señalar que las variables familiares analizadas podrían marcar en cierta forma la trayectoria de consumo en las participantes. El efecto de estas variables (escolaridad de padres, presencia de padre/madre, adicción familiar, etc.) las coloca en menor o mayor riego para adquirir la adicción a menor o mayor edad. La familia cumple varias funciones, principalmente la de aportar patrones de conducta, pautas de convivencia, modela sentimientos y establece vínculos familiares entre otros, que en conjunto pueden disminuir las conductas de riesgo. Las participantes provenían de escenarios inseguros en donde convivían con conflictos familiares, violencia, ausencia de padre/madre y con menor nivel socioeconómico, además de convivir en el mismo núcleo familiar con personas adictas. Este contexto las hace aún más vulnerables y propensas a un mayor riesgo de desarrollar conductas adictivas y problemas emocionales.

Es necesario hacer visibles a las mujeres en las políticas y programas de prevención de adicciones. Las adicciones en las mujeres son diferentes a las de los hombres en cuanto que en número son menos pero su incidencia tiende a incrementarse. A partir de la identificación de las trayectorias de consumo que aporta este trabajo, en los escenarios escolares se hace necesaria la detección temprana tanto de estudiantes como de familias en riesgo y el establecimiento de una unidad de consejería y canalización oportuna de casos. Esto se puede hacer a través de los departamentos de orientación escolar, tutorías o por medio de la capacitación al personal adscrito. Se propone establecer protocolos de actuación desde las instituciones escolares para dar seguimiento a los casos detectados incluyéndola 
promoción de un sistema más eficaz de comunicación con personas adultas significativas en la escuela independientemente del tipo de problemática personal o familiar por la que atraviesen la niña/niño/joven.

\section{REFERENCIAS}

Benítez J. (2018) Consumo de drogas en solicitantes de tratamiento en centro de Integración Juvenil. Drogas ilícitas. Segundo semestre de 2017. Sistema de Información Epidemiológica del Consumo de Drogas. Dirección de Investigación y Enseñanza Subdirección de Investigación. Secretaría de Salud. México. [fecha de consulta 20 de agosto de 2020]. Disponible en http://www.cii.gob.mx/Programas/Investigacion/pdf/18-01a.pdf

Blanco Zamora, P., Sirvent Ruiz, C. \& Palacios Ajuria, L. (2005). Diferencias de género en la adicción e implicaciones terapéuticas. Salud y drogas, 5(2), 81-97.[fecha de Consulta 13 de Abril de 2021]. ISSN: 1578-5319. Disponible en: https://www.redalyc.org/articulo.oa?id=83905205

Ivete, E. \& Estevéz, A. (2009). Consumo de drogas en adolescentes: El papel del estrés, la impulsividad y los esquemas relacionados con la falta de límites. Adicciones, 20(1), 49-56. [fecha de consulta 28 de agosto de 2020]. Disponible en: https://www.redalyc.org/articulo.oa?id=2891/289122882007

Cid-Monckton, P. \& Pedrão, L. (2011). Factores familiares protectores y de riesgo relacionados al consumo de drogas en adolescentes. Revista Latino-Americana de Enfermagem, 19, 738-745. [fecha de consulta 19 de julio de 2020]. Disponible en: https://www.redalyc.org/articulo.oa?id=2814/281421968010

ENCODAT (2017). Encuesta Nacional de Consumo de Drogas, Alcohol y Tabaco, 2016-2017. Coordinación de la Comisión Nacional contra las Adicciones. Instituto Nacional de Psiquiatría y el Instituto Nacional de Salud Pública. México.

Galaviz G. (2015) Mujeres, adicción y rehabilitación: Reflexiones desde la frontera noroeste de México. Salud Colectiva; 11(3):367-379.

Gutiérrez, M. \& Romero, I. (2014). Resiliencia, bienestar subjetivo y actitudes de los adolescentes hacia el consumo de drogas en Angola. Anales de psicología, 30 (2), 608-619.

Hernández-Serrano, O., Font-Mayolas, S., \& Gras, M. E. (2015). Policonsumo de drogas y su relación con el contexto familiar y social en jóvenes universitarios. Adicciones, 27 (3), 205-213. doi: http://doi.org/10.20882/adicciones.707

Guzmán F. Vargas J., Candia J, Rodríguez L. \& López K. (2018) Influencia de la presión de pares y facebook en actitudes favorecedoras al consumo de drogas ilícitas en jóvenes universitarios mexicanos. Health and Addictions, $19(1), 22-30$

Medina-Mora ME, Natera G \& Borges G. (2002). Alcoholismo y abuso de bebidas alcohólicas. Observatorio mexicano en tabaco, alcohol y otras drogas, 2002. México: Secretaría de Salud, Consejo Nacional contra las Adicciones; p. 15-25.

Padua J. (1996). Técnicas de investigación aplicadas a las Ciencias Sociales. México: Fondo de Cultura Económica, pp. 82-83.

Perasso G, Carraro E \& Ozturk Y (2020) Family mealtime against adolescents' drinking, drug use, and other risk behaviours. Then and now. Health and Addiction, 20(2), 119-134.

Pérez, C. (2011). Factores psicosociales asociados al uso de bebidas alcohólicas y tabaco en adolescentes tardíos de una Universidad privada en lima. Revista de Psicología, 23(2). https://doi.org/10.18800/psico.199502.003 
Pérez A. \& Correa M. (2011). Identidad femenina y consumo de drogas: un estudio cualitativo. Liberabit, 17(2), 211-222. [fecha de consulta 31 de agosto de 2020] Disponible en: http://www.scielo.org.pe/scielo.php?script=sci arttext\&pid=S1729-48272011000200010\&lng=es\&t|ng=es.

Pérez Gómez, A. \& Sierra Acuña, D. (2007). Recuperación natural y recuperación con tratamiento del consumo de drogas y alcohol. Adicciones, 19 (4), 409-421.

Romo N. \& Gil E. (2006). Género y uso de drogas. De la ilegalidad a la legalidad para enfrentar el malestar. Trastornos Adictivos, 8 (4), 243-250.

Sánchez L. (2009). Intervenciones preventivas sensibles a la perspectiva de género. Género y Drogas. Xunta de Galicia. [fecha de consulta 15 de junio de 2020] Disponible en: http://www.fundacioncsz.org/ArchivosPublicaciones/219.pdf

Sánchez Pardo L. (2014). Prevención del consumo de drogas con perspectiva de género. Recomendaciones con base en la evidencia. Diputación de Alicante. Área de Igualdad y Juventud. España. [fecha de consulta 15 de junio 2020] Disponible en: https://pnsd.sanidad.gob.es/profesionales/publicaciones/catalogo/bibliotecaDigital/ publicaciones/pdf/2014 Guia prevencion genero Diputacion Alicante.pdf

Serrano M. (2019) Caracterización de condiciones socioeconómicas de mujeres relacionadas con problemas de drogas: Las mujeres privadas de la libertad por delitos de drogas. Bogotá: Organización de las Naciones Unidas. [fecha de consulta 16 de junio de 2020] Disponible en: http://fileserver.idpc.net/library/Las\%20mujeres\%20privadas\%20de\%20la\%20liberta\%20por\%20delitos\%20de\%20drogasV2.pdf

Valenzuela M, Ibarra R., Zubarew A, Tamara G, \& Correa, I (2013). Prevención de conductas de riesgo en el Adolescente: rol de familia. Index de Enfermería, 22(1-2), 50-54. https://dx.doi.org/10.4321/S1132-12962013000100011

Velasco M., Hernández N, Herrero M, \& Hernández R. (2013) Las mujeres y las adicciones. Información estadística sobre el consumo de alcohol, tabaco y drogas en México. Centro de Estudios para el Adelanto de las Mujeres y la Equidad de Género. Cámara de Diputados LXII Legislatura. México, Julio, 2013.

WHO, ASSIST, Working Group (2002). The Alcohol, Smoking and Substance Involvement Screening Test (ASSIST): Development, reliability and feasibility Addiction, 97, pp. 1183-1194.

UNODC (2019) Informe Mundial sobre drogas 2019. ONUSIDA, OMS y Banco Mundial. [fecha de consulta 20 de junio 2020] Disponible en: https://wdr.unodc.org/wdr2019/prelaunch/WDR2019 B1 S.pdf 